\title{
Estado del arte \\ Acerca del proceso de adaptación a la escuela De los niños menores de 3 años
}

\author{
State of art \\ About the school adaptation process \\ In children under 3 years old \\ Laura Elena Burneo Farfán ${ }^{1}$ \\ Universidad San Ignacio de Loyola
}

Recibido: $27-11-19$

Aceptado: $17-12-19$

\section{Resumen}

El objetivo de este artículo es divulgar destacadas investigaciones que se han venido realizando durante los últimos diez años en el campo del Proceso de Adaptación a la Escuela de los niños menores de 3 años. Se trata de un periodo crítico al cual quedan expuestos nuestros niños dada la creciente participación de la mujer en el ámbito laboral. Este proceso explica la transición del niño de una vida hogareña con una atención personalizada de los padres o sustitutos rodeada de mucho afecto a un ambiente educativo.

En este trabajo se hace una revisión del tema dividiéndolo en cuatro factores que pueden determinar la calidad de dicha adaptación: la sobreprotección familiar al niño, el apego infantil, el cambio de ambiente y el rol de los padres. Se espera que esta data sea de utilidad para los investigadores interesados en explorar, describir, correlacionar, y explicar la dinámica socioemocional en la que se desenvuelve este interesante periodo en la vida de las personas.

Palabras clave: Adaptación; Escuela; Padres; Apego; Integración.

\begin{abstract}
IThe objective of this article is to disseminate outstanding research that has been carried out during the last ten years in the field of the School Adaptation Process for children under 3 years. This is a critical period to which our children are exposed given the increasing participation of women in the workplace. This process explains the child's transition from a home life with personalized attention from parents or substitutes surrounded by much affection for an educational environment.
\end{abstract}

In this work a review of the subject is made by dividing it into four factors that can determine the quality of such adaptation: family overprotection of the child, child attachment, change of

\footnotetext{
1 Docente, Universidad San Ignacio de Loyola. E-mail: laura.burneo@usil.pe

(C) Los autores. Este artículo es publicado por la Revista de Investigación en Psicología de la Facultad de Psicología, Universidad Nacional Mayor de San Marcos. Este es un artículo de acceso abierto, distribuido bajo los términos de la licencia Creative Commons Atribucion - No Comercia_Compartir Igual 4.0 Internacional. (http://creativecommons.org/licenses/by-nc-sa/4.0/) que permite el uso no comercial, distribución y reproducción en cualquier medio, siempre que la obra original sea debidamente citada.
} 
environment, and the role of parents. This data is expected to be useful for researchers interested in exploring, describing, correlating, and explaining the socio-emotional dynamics in which this interesting period unfolds in people's lives.

Keywords: Adaptation; School; Parents; attachment; Integration.

El período de adaptación escolar representa un gran reto social, afectivo y psicológico para el niño, durante este período es importante que exista un apoyo entre la institución educativa, las maestras y los padres (Albornoz, 2017). Este proceso puede ser definido como el período en el que el niño pasa de una unidad de convivencia muy pequeña, conocida e íntima (el hogar), a otra de un ámbito más amplio, extraño y público, (la escuela), donde los objetivos básicos son la socialización, la integración y la adaptación de los niños a la sociedad. Fomentar las habilidades sociales tempranas puede ser un objetivo importante para aumentar la adaptación positiva a la escuela para los niños pequeños, especialmente aquellos con algún déficit en su capacidad de aprendizaje (Mcintyre, Blacher \& Baker, 2006).

En cuanto al rol de los padres, investigaciones previas demuestran que la participación escolar de madres y padres de familia tiene un importante rol en la adaptación de sus hijos pequeños a la escuela (Tan \& Goldberg, 2008). No obstante, también observaron que las asociaciones entre los niveles de participación de los padres en la escuela y los resultados en la adaptabilidad del niño no siempre fueron positivas. Los hallazgos de estos investigadores resaltan la complejidad de la temprana participación de los padres en la escuela a la que van sus hijos y sus posteriores implicancias vinculadas a su desarrollo académico.

\section{Factores clave en el proceso de adaptación infantil a la escuela}

\subsection{La sobreprotección}

En un estudio llevado a cabo por Albornoz (2017) en la ciudad de Quito, Ecuador, se ha concluido que existe una relación directa entre sobreprotección y adaptación escolar, que se manifiesta esencialmente desde el punto de vista afectivo y psicológico por parte de los niños. Investigaciones llevadas a cabo en la Universidad de Santiago de Compostela (2019), concluyen que el proceso de adaptación es algo que el niño tiene que afrontar con los pocos recursos emocionales que tiene, pero al mismo tiempo es algo para lo que puede ser preparado, ayudado en conjunto por sus madres, padres y educadores. Por lo tanto, se trata de un período crítico en el que necesariamente se van a producir crisis emocionales de ambas partes: niños y adultos.

Siguiendo el Informe del Peaceful Parents Institute (2019), el estrés del cambio puede manifestarse de diferentes maneras para diferentes niños y acentuar 
sus pedidos de sobreprotección. Muchos niños se tornarán más melosos, pedirán más abrazos, y en general estarán solicitando mucha más atención por parte de sus adultos significativos. Hay también casos de niños que empiezan a volverse más vulnerables a los accidentes caseros y naturalmente también a sucesos sorpresivos en sus escuelas. Los niños pequeños pueden haber estado ya entrenados a ir al baño sin temor, pero esta habilidad puede involucionar justo a raíz del incremento de una inconsciente sobreprotección paternal. Estudios llevados a cabo por la Universidad de Santiago de Compostela (2019), concluyen que la posible rebeldía de los niños y, a veces, actitudes violentas contra sus padres son una protesta contra la situación que los separó de ellos. Tal separación conlleva en los niños sobreprotegidos acentuados sentimientos de miedo, resentimiento, y frustración.

Whitebread (2013) investigador de la Universidad de Cambridge, aboga por una "extensión de la vida informal" del preescolar (basada en los juegos), y está a favor de un retraso en el inicio de la 'escolarización' formal. Expone además buenos resultados académicos, motivacionales y de bienestar alcanzados por los niños que habían asistido a programas preescolares basados en juegos, planteados o creados por los propios niños. En resumen, Whitebread afirma que un período extendido de educación preescolar basada en juegos de alta calidad es un factor de mucha ayuda para la adaptación de los niños a su primera escuela, especialmente de aquellos niños provenientes de hogares disfuncionales.

Cooper (2019) resalta el importante papel que desempeña la rutina en esta nueva adaptación. Observa que las rutinas ayudan al niño a tener un cierto nivel de control de su día y lo ayudan a saber lo que vendrá después. Como de todos modos la ansiedad de la separación es un periodo difícil para el niño y también para sus padres, saber que se tiene una agenda diaria por cumplir, les permite a ambos superar los iniciales periodos de incertidumbre.

\subsection{El apego infantil}

Este fuerte vínculo sentimental específico y especial que se forma entre el niño y sus padres (especialmente la madre), es para Bossi, Brites \& Piccinini (2017) una variable que, si llega a tener un excesivo protagonismo, terminará por perturbar la adaptación del niño a la escuela. Por un lado, la madre tendrá sentimientos de culpa, de inseguridad, al tener que dejar al hijo pequeño en un lugar ajeno a su hogar. Por su parte, el niño tendrá fuertes sentimientos de desamparo, abandono, y miedo a lo desconocido. La escuela es un mundo desconocido para los niños pequeños, quienes nunca antes la habían visto y en la que ahora deberán pasar buena parte de sus vidas (Temas para la Educación, 2011). Es por eso muy importante la estabilidad del microsistema familiar para afianzar el buen desempeño escolar de los niños pequeños. Afirman Menéndez, Jiménez \& Lorence (2010) que los hogares que funcionan como contextos promotores del desarrollo infantil potencian, a su vez, 
la adaptación de los menores al entorno escolar en aspectos que van más allá del rendimiento académico.

Por tal razón los investigadores continúan enfatizando hasta hoy que es fundamental un previo trabajo de adaptación socioemocional tanto para los padres como para los niños. Mueller (2018) observa que incluso los niños extravertidos, pero con fuertes sentimientos de apego, pueden tener problemas serios para adaptarse a una nueva escuela de inmediato. Es por eso que los padres de los niños pequeños deben estar siempre dispuestos a prestarle atención, pero solamente cuando sea realmente necesario, teniendo conciencia que establecerse fuera del hogar lleva tiempo.

\subsection{El cambio de ambiente}

Desde los 12 hasta los 18 meses el niño vive un período particularmente sensible para adaptarse a su entorno (Poole, Miller \& Church, 2018). Los niños pequeños pueden estar muy conscientes de la existencia de varios entornos, pero eso no garantiza su adaptabilidad emocional, puesto que su lenguaje, memoria y habilidades de afrontamiento aún se están desarrollando. afirmaban que, si bien los maestros de preescolar sugieren que lo fundamental para la adaptación del niño a su primera escuela es el apoyo de sus padres, los padres a su vez señalan que lo más importante en este proceso es el funcionamiento general de la escuela, y las características y metodología utilizada por las profesoras de Educación Inicial (Correia \& Marques, 2016)

En un estudio llevado a cabo por Ackinar (2013) se concluía que -de acuerdo a los resultados que obtuvo- un mayor estatus socioeconómico, mejores estrategias adaptativas de disciplina parental, y mayores niveles de competencia socioconductual en los niños, conducen a una mejor adaptación escolar. En este sentido, Ackinar asevera que la adaptación saludable a la escuela incluye tanto la adaptación cognitiva como la social. Sobre el hecho del cambio de ambiente, Mueller (2018) sostiene que los niños tienen una visión limitada del mundo, y algo que a los adultos podría parecer "una ridiculez" como no saber orientarse en los ambientes de su escuela (aula, servicios higiénicos, puerta de salida, patio de juegos) para los niños son grandes y muy serios problemas que incluso los pueden llevar a no desear salir más de sus hogares. Al respecto, Menéndez, Jiménez, \& Lorence, (2010) llegaron también a la conclusión de que las implicaciones negativas para el desarrollo de los niños que crecen en esos entornos negativos trascienden las paredes de sus hogares y repercuten en su adaptación escolar desde muy temprano. Las autoras del estudio Familia y adaptación escolar durante la infancia, afirman que hay suficiente evidencia acerca del rol acumulativo e intensificador que parece jugar el paso del tiempo. 
Por su parte, Ackinar (2013) encontró que un mayor estatus socioeconómico, estrategias adaptativas de disciplina parental y mayores niveles de competencia socioconductual en los niños, conduce a una mejor adaptación escolar. La buena adaptación del niño pequeño a la escuela comprende no solamente su adaptación cognitiva, sino básicamente la social. Aunque no se encontró un claro vínculo entre el desarrollo socioconductual del niño y su adaptación escolar hay evidencias de que las variables cognición y desarrollo socioemocional, son factores críticos y decisivos para un buen arraigo en las aulas.

\subsection{El rol de los padres}

Borbélyová (2017) realizó un estudio en el que abordaba el tema de la adaptación escolar. Examinó los aspectos individuales de la adaptación en la educación primaria temprana, prestando especial atención a los problemas de adaptación y su prevención. El objetivo de su investigación fue proporcionar información sobre el enfoque contemporáneo del problema y presentar algunas estrategias que pudieran aplicarse en la práctica para reducir la incidencia de problemas de adaptación. Concluye que el período de adaptación en la educación primaria temprana es un momento muy exigente en la vida del niño. Durante su proceso de adaptación, el niño necesita la ayuda de los adultos para sobreponerse con éxito a esta crisis.

Tabla

Cronología de investigaciones: Adaptación a la Escuela en niños menores de 3 años.

\begin{tabular}{|c|c|c|c|c|}
\hline \multicolumn{5}{|c|}{ FACTORES CENTRALES ESTUDIADOS } \\
\hline $\begin{array}{c}\text { Años } \\
\boldsymbol{V}\end{array}$ & SOBREPROTECCIÓN & APEGO INFANTIL & $\begin{array}{l}\text { CAMBIO DE } \\
\text { AMBIENTE }\end{array}$ & $\begin{array}{l}\text { ROL DE LOS } \\
\text { PADRES }\end{array}$ \\
\hline 2010 & & $\begin{array}{l}\text { Menéndez, Jiménez \& } \\
\text { Lorence }\end{array}$ & $\begin{array}{l}\text { Menéndez, Jiménez } \\
\text { \& Lorence }\end{array}$ & \\
\hline 2011 & & $\begin{array}{l}\text { Revista Temas para la } \\
\text { Educación }\end{array}$ & & \\
\hline 2012 & & & & \\
\hline 2013 & Whitebread & & Ackinar & \\
\hline 2014 & & & & \\
\hline 2015 & & & & \\
\hline 2016 & & & $\begin{array}{l}\text { Correia \& Marques- } \\
\text { Pinto }\end{array}$ & $\begin{array}{l}\text { Correia \& } \\
\text { Marques-Pinto }\end{array}$ \\
\hline 2017 & Albornoz & $\begin{array}{l}\text { Bossi, } \\
\text { Brites \& Piccinini }\end{array}$ & & $\begin{array}{l}\text { Borbélyová } \\
\text { Bossi, Brites \& } \\
\text { Piccinini }\end{array}$ \\
\hline 2018 & & Mueller & $\begin{array}{l}\text { Poole, Miller, \& } \\
\text { Booth Church } \\
\text { Mueller }\end{array}$ & \\
\hline 2019 & $\begin{array}{l}\text { Universidad } \\
\text { De Santiago de Compostela. } \\
\text { Peaceful Parents Institute } \\
\text { Cooper }\end{array}$ & & & \\
\hline
\end{tabular}


Por su parte, Correia \& Marqueso (2016) establecen que hay una serie de factores internos y externos asociados con la buena o mala adaptación del niño pequeño a la escuela, pero que también hay estrategias tanto para las familias como para la escuela, que pueden facilitar dicha adaptación. En cuanto al rol de la madre, Bossi, Brites \& Piccinini (2017) establecen que hay una complejidad de variables que van a favorecer o no la adaptación de sus hijos. Entre ellas destacan: el nivel de seguridad que tienen las madres sobre la adaptación de sus hijos, la confianza en la guardería, en cuanto al espacio físico, y los materiales lúdicos.

\section{DISCUSIÓN}

Se aprecia que en la presente década la investigación sobre el tema aquí tratado se ha centrado principalmente en el apego infantil y el cambio de ambiente en la cual se prioriza que la dinámica familiar estimulante del desarrollo autónomo en el niño favorece mayores recursos adaptativos, así como el nivel de participación de los padres en la escuela (Menéndez, Jiménez, \& Lorence, (2010) a diferencia de lo que hoy se tiene: investigaciones que mas bien se orientan a estudiar el factor de la sobreprotección del niño pequeño ad portas de iniciar el largo camino de su escolaridad (Universidad de Santiago de Compostela, 2019). No obstante, cada uno de los cuatro factores expuestos y estudiados han tenido su momento de auge, en el que fueron considerados como el elemento más importante dentro de la problemática de la adaptación del niño de alrededor de tres años a la escuela.

En todos los estudios realizados por los autores aquí registrados, hay un denominador común: esta fase de la vida infantil es un periodo de crisis psicológica, social, y emotiva del niño (Bossi, Brites \& Piccinini, 2017). Es el paso decisivo que da el ser humano de la sociedad contemporánea de su entorno más íntimo (sus padres y hermanos) a un entorno ilimitado (la sociedad, la cultura, la educación, la convivencia con el mundo). En cada uno de los estudios revisados destaca el rol crucial que le compete a los padres, a los docentes, y al sistema educativo en general (Correia \& Marques, 2016; Bossi, Brites \& Piccinini, 2017). Es por ello la importancia que tiene el hecho de contar con sistemas educativos actualizados, de buena calidad, democráticos, e integradores.

Así mismo, es necesario contar con padres de familia no solo mejor preparados para guiar, educar y criar a su descendencia, sino además dispuestos a cambiar su escala de valores muchas veces rígida e intolerante (Borbélyová, 2017). Finalmente, la escuela debe ser cada vez más acogedora, abierta, integradora, y ante todo solvente en cuanto a su calidez humana (Correia \& Marques, 2016)

\section{CONCLUSIONES}

1. El período de adaptación escolar representa un gran reto socioemocional para el niño, al ser una etapa que marca el tránsito infantil de una unidad 
de convivencia muy pequeña, conocida, e íntima (el hogar), a otra de un ámbito más amplio, extraño y público, (la escuela),

2. La activa participación de madres y padres de familia tiene un rol decisivo en la adaptación de sus hijos pequeños a la escuela.

3. Existe una relación directa entre sobreprotección y adaptación escolar, que se manifiesta esencialmente como acentuados sentimientos de miedo, resentimiento, y frustración por parte del niño.

4. Un período extendido de educación preescolar basada en juegos que promuevan su motivación, necesidad de explorar, constituyen un factor de mucha ayuda para la adaptación de los niños a su primera escuela.

5. Es fundamental un previo trabajo de adaptación socioemocional tanto para los padres como para los niños, siendo la escuela la promotora y guía de dicha tarea.

6. Los maestros de preescolar sugieren que lo fundamental para la adaptación del niño es el apoyo de sus padres, pero los padres a su vez señalan que lo más importante en este proceso es la escuela.

7. El período de adaptación escolar es un momento muy exigente en la vida del niño pequeño, por lo que necesita la ayuda de los adultos para salir con éxito de esta crisis.

\section{REFERENCIAS}

Ackinar, B. (2013) Los predictores de la adaptación escolar en la primera infancia. Procedia: Social and Behavioral Sciences, 93, 1099-1104. doi: 10.1016/j.sbspro.2013.09.338

Albornoz J. (2017). La adaptación escolar en los niños y niñas con problemas de sobreprotección. Universidad y Sociedad, 9(4), 177-180. Recuperado de http://rus. ucf.edu.cu/index.php/rus

Borbélyová, D. (2017). Adaptation to the School Enviroment in Early Elementary Education. Journal of education in contexts. 2(13), 206-228. Recuperado de https:// www.paidagogos.net/issues/2016/2/article.php?id=13

Bossi, Brites y Piccinini (2017). Adaptación a la Guardería: Factores que Facilitan o no este Período. Paideia, 1(27), 448-456. doi: 10.1590/1982-432727s1201710

Cooper, S. (2019) Cómo tratar la ansiedad de separación en preescolares. Enseñando a niños de 2 y 3 años. Recuperado de https://teaching2and3yearolds.com/how-to-dealwith-separation-anxiety-in-preschool/

Correia, K. y Marques, A. (2016) Adaptación en la transición a la escuela: perspectivas de los padres, maestros de preescolar y primaria. Educational Research, 58(3), 247-264, doi: 10.1080/00131881.2016.1200255 
Federación de Enseñanza de CC.OO. de Andalucía. (2011). El Apego en la Educación Infantil. Recuperado de https://www.feandalucia.ccoo.es/docu/p5sd8334.pdf

Mcintyre, Ll, Blacher J., \& Baker, B. (2006). The transition to school: adaptation in young children with and without intellectual disability. PubMed 50(5), 349-361. Recuperado de https://www.ncbi.nlm.nih.gov/pubmed/16629928

Menéndez, S., Jiménez, L., y Lorence, B. (2010). Familia y adaptación escolar durante la infancia. Revista de Educación, 10, 97-110. Recuperado de http://rabida.uhu.es/ dspace/bitstream/handle/10272/2146/b15480045.pdf?sequence $=1$

Mueller, L. (2018) Cómo ayudar a los niños a adaptarse a una nueva escuela. Moving. com Recuperado de https:/www.moving.com/tips/how-to-help-kids-adapt-to-anew-school/

Peaceful Parents Institute (2019). Ayudando a los niños a adaptarse al cambio. Recuperado de https://www.peacefulparent.com/helping-children-adapt-to-change/

Plan de Adaptación para niños y niñas de 3 años. (2010). Recuperado de https:// orientacionandujar.files.wordpress.com/2010/09/plande-adoptacion-3-anos.pdf

Poole, C., Miller, S., y Church, E. (2018). Ages \& Stages: How Children Adjust to School. Recuperado de https://www.scholastic.com/teachers/articles/teaching-content/agesstages-how-children-adjust-school/

Tan, E. \& Goldberg, W. (2008). Parental school involvement in relation to children's grades and adaptation to school. Journal of Applied Developmental Psychology 30(4), $442-$ 453. Recuperado de https://www.researchgate.net/publication/222576873_Parental_ school_involvement_in_relation_to_children's_grades_and_adaptation_to_school

Temas para la Educación (2011) El apego en Educación Infantil. Federación de enseñanza de CC.OO. de Andalucía, España. ISNN 1989-4023 Recuperado de https://www. feandalucia.ccoo.es/docu/p5sd8334.pdf

Universidad de Santiago de Compostela (2019) El periodo de adaptación. Recuperado de http://www.usc.es/en/servizos/breogan/adaptacion.html

Whitebread, D. (2013) Edad escolar inicial: la evidencia. Research. University of Cambridge. Recuperado de https://www.cam.ac.uk/research/discussion/schoolstarting-age-the-evidence 